PUBLIC HEALTH RESEARCH

\title{
Characterization of Diabetic Nephropathy and Its Correlates in a Selected Outdoor-Based Diabetic Clinic in Rangpur, Bangladesh
}

\author{
Most. Zannatul Ferdous ${ }^{1}$, Md. Abdur Rahman ${ }^{1}$, Md. Ruhul Furkan Siddique ${ }^{1}$, Md Abdul Halim ${ }^{2}$ and Sheikh \\ Jafia Jafrin ${ }^{3}$ \\ ${ }^{1}$ Department of Public Health and Informatics, Jahangirnagar University, Savar, Dhaka-1342 Bangladesh. \\ ${ }^{2}$ Research \& Development Analytical, Incepta Pharmaceutical Ltd. \\ ${ }^{3}$ Department of Geological Sciences, Jahangirnagar University, Savar, Dhaka-1342. \\ *For reprint and correspondence: Most. Zannatul Ferdous, Department of Public Health and Informatics, \\ Jahangirnagar University, Savar, Dhaka-1342, Bangladesh. \\ Email: m.zannatul.ferdous@juniv.edu,ferdousphhub@gmail.com
}

\section{ABSTRACT}

\begin{tabular}{|c|c|}
\hline Introduction & $\begin{array}{l}\text { Diabetes mellitus (DM) is a global public health problem. DM is associated with } \\
\text { the rising burden of diabetic complications. Diabetic nephropathy (DN) is one of } \\
\text { the life-threatening and irreversible microvascular complications of DM. }\end{array}$ \\
\hline Methods & $\begin{array}{l}\text { A cross-sectional study was conducted from November } 2017 \text { to April } 2018 \text {. This } \\
\text { study aimed to characterize the proportion of DN among people living with } \\
\text { diabetes ( } \mathrm{n}=290 \text {, where } 40 \text { T1DM, } 200 \text { T2DM as cases group and } 50 \text { nondiabetic } \\
\text { as a control group) and the factors associated with it. The sample was selected } \\
\text { using convenience sampling. Data were collected using structured questionnaire } \\
\text { and analyzed by SPSS-22. Stages of DN were classified according 'Revised, } \\
\text { Classification of DN' given by the 'Joint Committee on Diabetic Nephropathy', } \\
\text { Japan, 2014. }\end{array}$ \\
\hline Results & $\begin{array}{l}\text { The proportion of pre-nephropathy (PN), incipient nephropathy (IN) and overt } \\
\text { nephropathy (ON) was } 10 \%, 82.5 \% \text { and } 7.5 \% \text { respectively in T1DM. Similarly, } \\
\text { in T2DM the proportion of PN, IN, ON and chronic kidney failure (CKF) was } \\
5.5 \% 81 \%, 10 \% \text {, and } 3.5 \% \text { respectively. Irrespective of diabetic group, according } \\
\text { to multivariate analysis, older age (adjusted OR }=1.05 \text {, CI: } 1.01-1.08 \text {; adjusted } \\
\text { OR: } 2.33 \text {, CI: } 2.01-2.99 \text { ), and female sex (adjusted OR }=0.39 \text {, CI: } 0.19-0.77 \text { ) were } \\
\text { independently associated with DN adjusting BMI, SBP, DBP and FBS level. }\end{array}$ \\
\hline Conclusions & $\begin{array}{l}\text { This study showed the proportion of DN was high among diabetic and mostly in } \\
\text { type } 2 \text { diabetics with severe stage. Older age, and female sex were independently } \\
\text { associated with DN. }\end{array}$ \\
\hline Keywords & $\begin{array}{l}\text { Type } 1 \text { diabetes - type } 2 \text { diabetes - diabetic nephropathy - estimated glomerular } \\
\text { filtration rate - Albumin to Creatinine ratio - Bangladesh. }\end{array}$ \\
\hline
\end{tabular}

Article history:

Received: 14 April 2020

Accepted: 1 June 2021

Published: 1 September 2021 


\section{INTRODUCTION}

Diabetes mellitus (DM) is one of the most common and rapidly increasing chronic diseases globally, and has been called a "diabetes epidemic". DM is associated with many long term complications like diabetic nephropathy (DN) which represents the most common cause of ESRD (end-stage renal disease) worldwide accounting for the high mortality rate in patients with diabetes. ${ }^{2,3}$ As a result, diabetes is the leading cause of kidney failure worldwide also and is responsible for approximately 6 percent of total global mortality. ${ }^{4}$ If diabetes is not treated early and adequately, many patients will reach advanced and irreversible DN. ${ }^{5}$ Evidence suggests that type 1 diabetes starts at a younger age among Asian people compared to Caucasians and those genetic factors and lifestyle risk factors are more common in Asian people. Also, as compared with Caucasians, South Asians have a 3- fold greater risk of developing DN and an almost 40-fold greater risk of developing $\mathrm{DN}$, possibly due to a higher proportion of insulin resistance in the latter. ${ }^{6}$ It affects $20-40 \%$ of type 1 and type 2 diabetic patients. ${ }^{15}$ However, there is strong evidence that a number of interventions if initiated at early stage of diabetic kidney disease reduce the risk and slow the progression of kidney damage. ${ }^{7}$ Though there are many studies exist in different country for determining proportion rate of DN whereas there is hardly any national data of DN in Bangladesh, a clinic based case-control study reported one third of newly detected diabetic patients having DN. ${ }^{8}$ So, the objective of this study was to assess the proportion of DN in Bangladeshi diabetic population who receiving services from an outdoor-based diabetic clinic and its associated factors.

\section{METHODS}

Study design and population

This descriptive type of cross-sectional study was conducted at diabetic clinic, in Rangpur, Bangladesh from November 1, 2017 to April 29, 2018.

The patients attending study area (diabetic clinic) fulfilling the inclusion criteria were selected conveniently as the study participants.

\section{Sample size determination}

The sample size was calculated using the following equation (1):

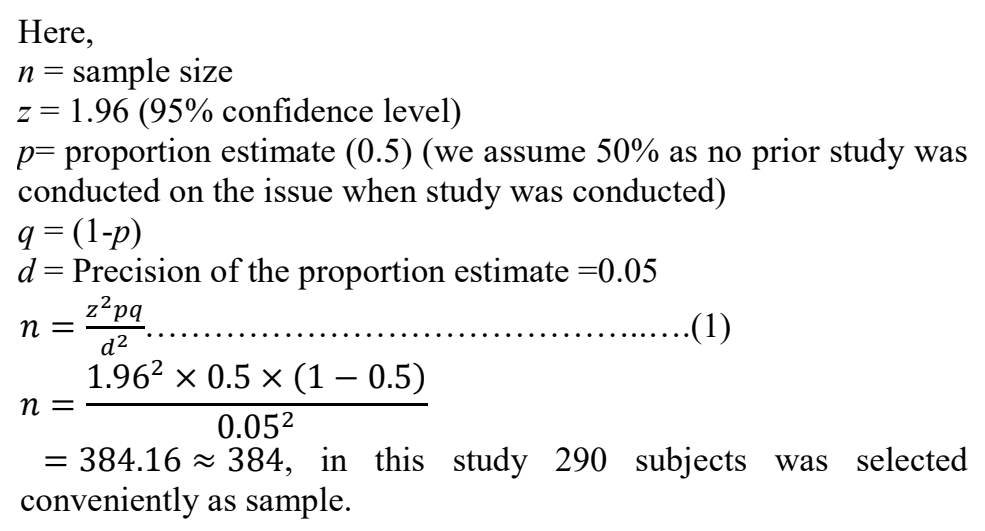

\section{Inclusion criteria}

The inclusion criteria were adult subjects (male and female) aged $20-79$ years and voluntarily agreed to participate in the study as well as to provide written informed consent.

\section{Exclusion criteria}

The exclusion criteria included i) unable to provide informed consent ii) co-morbid diseases such as infection, major surgery, malabsorption, cancer and those undergoing dialysis as well as renal transplantation iii) concomitant acute or chronic severe diseases such as renal failure, hepatic insufficiency iv) Pregnant female v) Very old, disabled patient bid-ridden or mentally incompetent subjects were excluded vi) heavily exercised ( more than 1 hour of vigorous exercise on a day of the collection period).

\section{Selection of the case and control}

The patients who went to the diabetic clinic (Rangpur Diabetic Association, an outdoor-based diabetic clinic) for regular checkup were the target group for the case, of them who had suffered from diabetes more than 4 years selecting conveniently as case sample. The case group either type 1 or type 2 diabetes patients was confirmed from their diabetic record book. The apparently healthy staff and relatives of the case group were targeted as control, of them who had ACR: $<30 \mathrm{mg} / \mathrm{g}$ selecting as control sample.

Data collection tools and technique

Data was collected through face-to-face interview. A structured questionnaire was used to collect data. The attending physicians, consultants and authority were informed about the research objectives, procedures, and inclusion and exclusion criteria. 
Measurements of the study variables

We collected data on socio demographic variables, blood pressure, weight, height and body mass index (BMI), duration of diabetes, habit of smoking. Fasting blood sugar (FBS) and serum creatinine level, urine albumin and creatinine for albumin to creatininie ratio (ACR) were measured in laboratory. Further, the duration of diabetes and blood pressure was obtained from diabetic records book. Morning urine sample was used to calculate albumin to creatinine ratio (ACR) in $\mathrm{mg} / \mathrm{g}$. Micro albumin was carried out using ELISA assay and creatinine was done by calorimetric methods using Fortress kit. Blood samples were obtained for test of serum glucose, serum creatinine. FBS was measured by enzymatic colorimetric (GOD-POD) method in the Humalyzer 3000. Estimation of sCreatinine was done by modified Jaffe method in the humalyzer 3000. Stages of DN were classified according to the guideline of "The Revised Classification of DN" given by the Joint Committee on Diabetic Nephropathy (JCDN), Japan, 2014. ${ }^{9}$

Data analysis and management

Data were analyzed using SPSS-22. Results were presented as mean and standard deviation (SD) for continuous variables, and as frequencies and proportions for categorical variables; $t$-test was used for continuous variables and chi square test for categorical variables. We conducted a Chi-square test as well as one way ANOVA followed by posthoc $L S D$ for the measures of association. A p-value $<0.05$ was used as a level of significance with a $95 \%$ confidence level.

\section{Ethical issues}

Study was approved by the institutional ethics committee. Precaution was taken to address the ethical aspect at the time of sample collection. Consent was obtained from the volunteers to participate study. Further ensuring that the identity of the individual would not be disclosed and the volunteers would be communicated involving any further analysis.

\section{RESULTS}

Characteristics of the study subjects

Table 1: There were a total of two hundred ninety study subjects', [forty T1DM subjects and two hundred T2DM subjects and fifty controls] where 138 were male and 152 were females. Out of these 25 males and 25 females were in control group, 21 males and 19 females were T1DM and 92 males and 108 female were T2DM. In T1DM and T2DM the mean ages were 21.8 years $(\mathrm{SD}=2.3)$ and 55.0 years $(\mathrm{SD}=9.4)$ respectively whereas controls were 29.9 years $(\mathrm{SD}=7.7)$. Only $29.4 \%$ had completed an undergraduate degree and $21.4 \%$ higher education or post-graduate degree in T2DM whereas in T1DM no one have competed higher education $(0.0 \%)$ and only $11.8 \%$ had undergraduate degree.

\section{Stages of diabetic nephropathy}

The proportion of DN was presented in Table 1 . In T1DM subjects, the proportion of the IN or microalbuminuria $33(82.5 \%)$ was the highest (eGFR: $\geq 30$, Microalbuminuria i.e; ACR:30$299 \mathrm{mg} / \mathrm{g})$ followed by PN with $4(10 \%)$ [eGFR: $\geq 30$, Normoroalbuminuria i.e; ACR: $<30 \mathrm{mg} / \mathrm{g}]$ ON or macroalbuminuria with $3(7.5 \%)$ [eGFR: $\geq 30$, Macroalbuminuria i.e; ACR: $\geq 300 \mathrm{mg} / \mathrm{g}$ ]. In T2DM subjects the highest percentage in IN with $162(81 \%)$ [eGFR: $\geq 30$, Microalbuminuriai.e; ACR:30$299 \mathrm{mg} / \mathrm{g}$ ] followed by ON with 20 (10\%) [eGFR: $\geq 30$, Macroalbuminuriai.e; ACR: $\geq 300 \mathrm{mg} / \mathrm{g}$ ], PN with $\quad 11 \quad(5.5 \%) \quad[\mathrm{eGFR}: \geq 30$ ,Normoroalbuminuriai.e; ACR: $<30 \mathrm{mg} / \mathrm{g}]$ and CKF with 7 (3.5\%) [Any status of Albuminuria i.e; ACR: $30-299 \mathrm{mg} / \mathrm{g}$ or $\geq 300 \mathrm{mg} / \mathrm{g}$; eGFR: $<30]$.

Table 1 Characteristics of the study subjects

\begin{tabular}{crrrrr}
\hline & \multicolumn{2}{c}{ Socio demographic characteristics Chi-square } & & \multicolumn{1}{c}{ Total } & P-value \\
Variables & Controls & T1DM & \multicolumn{1}{c}{ T2DM } & 0.703 \\
Sex (\%) & & & & & \\
Male & $25(18.1)$ & $21(15.2)$ & $92(66.7)$ & $138(47.59)$ & $\leq 0.001^{*}$ \\
Female & $25(16.4)$ & $19(12.5)$ & $108(71.1)$ & $152(52.41)$ & $\leq 0.001$ \\
Age (mean with SD) & $29.98 \pm 7.7$ & $21.8 \pm 2.3$ & $55.0 \pm 9.4$ & $46.1 \pm 15.9$ & \\
Marital status (\%) & & & & & \\
Married & $25(10.9)$ & $8(3.5)$ & $196(85.6)$ & $229(79.0)$ & $\leq 0.001$ \\
Unmarried & $23(41.8)$ & $31(56.4)$ & $1(1.8)$ & $55(19.0)$ & \\
Divorced & $2(33.3)$ & $1(6.7)$ & $3(50.0)$ & $6(2.1)$ & \\
Education (\%) & & & & & \\
Illiterate & $8(14.0)$ & $1(1.8)$ & $48(84.2)$ & $57(19.8)$ & \\
Primary & $0(0.00)$ & $12(17.1)$ & $58(82.9)$ & $70(24.1)$ & \\
Secondary & $9(16.4)$ & $4(7.3)$ & $42(76.4)$ & $55(19.0)$ & \\
Higher secondary & $12(18.2)$ & $21(31.8)$ & $33(50.0)$ & $66(22.8)$ \\
Undergraduate degree & $10(58.8)$ & $2(11.8)$ & $5(29.4)$ & $17(5.9)$ & $14(4.8)$
\end{tabular}




\begin{tabular}{|c|c|c|c|c|c|}
\hline Read Arabic & $0(0.00)$ & $0(0.00)$ & $4(100.0)$ & $7(1.4)$ & \\
\hline Only can sign & $0(0.00)$ & $0(0.00)$ & $7(100.0)$ & $49(2.4)$ & \\
\hline Smoking & & & & & $\leq 0.001$ \\
\hline Yes & $33(37.8)$ & $7(15.6)$ & $21(46.7)$ & $45(15.5)$ & \\
\hline No & $33(13.5)$ & $33(13.5)$ & $179(73.1)$ & $245(84.5)$ & \\
\hline Occupation (\%) & & & & & $\leq 0.001$ \\
\hline Farmer & $2(8)$ & $2(8)$ & $21(84)$ & $25(8.6)$ & \\
\hline Govt.job & $4(12.5)$ & $1(3.1)$ & $27(84.4)$ & $32(11)$ & \\
\hline Private job & $23(59)$ & $1(2.6)$ & $15(38.5)$ & $39(13.4)$ & \\
\hline Business & $4(8.7)$ & $6(13)$ & $36(78.3)$ & $46(15.9)$ & \\
\hline Housewife & $9(7.9)$ & $10(8.8)$ & $95(83.3)$ & $114(39.3)$ & \\
\hline Daily wages/Labor & $0(0.00)$ & $0(0.00)$ & $6(100.0 \mid)$ & $6(2.1)$ & \\
\hline Student & $8(21.6)$ & $0(0.00)$ & $20(81.4)$ & $28(9.7)$ & \\
\hline Monthly income (\%) & & & & & $\leq 0.001$ \\
\hline$\leq 15000$ & $37(17.8)$ & $14(6.7)$ & $157(75.5)$ & $208(71.1)$ & \\
\hline $15001-20000$ & $9(32.1)$ & $6(21.4)$ & $13(46.4)$ & $28(9.7)$ & \\
\hline$\geq 20001$ & $4(7.4)$ & $20(37.0)$ & $30(55.6)$ & 54 (18.6) & \\
\hline Monthly income (in BDT) & $12740 \pm 7850$ & $19326 \pm 6669$ & $11837 \pm 7551$ & $13026 \pm 7889$ & $\leq 0.001^{*}$ \\
\hline Family size $($ mean $\pm \mathrm{SD})$ & $4.88 \pm 1.66$ & $6.30 \pm 1.95$ & $5.07 \pm 2.18$ & $5.21 \pm 2.11$ & $0.002 *$ \\
\hline $\begin{array}{c}\text { Duration of diabetes } \\
\text { (years) }\end{array}$ & - & $8.03 \pm 2.82$ & $7.47 \pm 3.02$ & $7.56 \pm 2.90$ & $0.04 * *$ \\
\hline \multicolumn{6}{|c|}{ Biochemical and physiological characteristics of the study subjects } \\
\hline FBS (mmol/L) & $4.55 \pm 0.58$ & $6.81 \pm 0.87$ & $7.98 \pm 3.25$ & $7.24 \pm 3.01$ & $0.001 *$ \\
\hline sCreatinine $(\mu \mathrm{mol} / \mathrm{L})$ & $61.46 \pm 6.86$ & $66.95 \pm 8.39$ & $82.35 \pm 32.02$ & $88.84 \pm 33.23)$ & $0.002^{* \mathrm{~T}} \leq 0.001^{* \mathrm{~T}}$ \\
\hline eGFR (mL/min) & $110.54 \pm 13.2$ & $108.50 \pm 15.4$ & $72.73 \pm 22.6$ & $84.18 \pm 26.67$ & $0.517^{* \mathrm{~T}} \leq 0.001^{* \mathrm{~T}}$ \\
\hline $\mathrm{SBP}(\mathrm{mm} \mathrm{Hg})$ & $118.20 \pm 3.88$ & $120.25 \pm 8.61$ & $133.13 \pm 15.94$ & $128.78 \pm 15.16$ & $\leq 0.001 *$ \\
\hline DBP (mm Hg) & $79.20 \pm 1.82$ & $80.63 \pm 5.56$ & $84.74 \pm 6.22$ & $83.22 \pm 6.06$ & $\leq 0.001 *$ \\
\hline BMI $\left(\mathrm{kg} / \mathrm{m}^{2}\right)$ & $23.76 \pm 2.55$ & $21.99 \pm 2.68$ & $24.12 \pm 3.60$ & $23.77 \pm 3.39$ & $0.001 *$ \\
\hline \multicolumn{6}{|l|}{ Categories of DN } \\
\hline PN & - & $4(10)$ & $11(5.5)$ & $15(6.3)$ & \\
\hline IN & - & $33(82.5)$ & $162(81.0)$ & $195(81.3)$ & \\
\hline ON & - & $3(7.5)$ & $20(10.0)$ & $23(9.6)$ & 0.02 \\
\hline CKF & - & 0 & $7(3.5)$ & $7(2.9)$ & \\
\hline TKF & - & 0 & 0 & 0 & \\
\hline
\end{tabular}

Data are expressed as mean \pm SD, percentages in parentheses; Fisher Exact Test; Sig. (2-sided); * One way ANOVA analysis; ** Independent 2 samples test

- ${ }^{\mp}$ and ${ }^{\mp \top}$ indicated type $1 \mathrm{DM}$ vs controls and type $2 \mathrm{DM}$ vs controls respectively

- FBG Fasting Blood Glucose; sCreatinine Serum Creatinine; eGFR Estimated Glomerular filtration Rate; BMI Body Mass Index; SBP Systolic Blood Pressure; DBP Diastolic Blood Pressure, PN Pre nephropathy; IN Incipient Nephropathy; ON Overt Nephropathy; CKF Chronic Kidney failure; TKF Terminal kidney Failure

In case of T1DM, from the post- hoc LSD multiple comparison test it was assumed that there was a significant association between control vs $\mathrm{PN}$, $(p=0.005)$ and control vs ON $(p=\leq 0.001)$ on the basis of age (Table 2). In case of control vs IN $(p=\leq 0.001,0.001,0.029)$, show significance association on the basis of age, BMI and DBP respectively. Further, in case of $\mathrm{IN}$ and $\mathrm{ON}$ $(p=0.045)$ show significant association on the basis of SBP. In case of T2DM, different stages of DN: $\mathrm{PN}, \mathrm{IN}, \mathrm{ON}$ and CKF have a significant association $(p=\leq 0.001, \leq 0.001, \leq 0.001)$ with age, SBP, DBP but there were no significant association with BMI $(\mathrm{p}=0.862)$.

On the other hand using same test it was assumed that there was a significant association between control vs PN, $(p=\leq 0.001, \leq 0.001)$; controls vs IN ( $\mathrm{p}=\leq 0.001,0.044)$; controls and $\mathrm{ON}$ $(p=\leq 0.001, \leq 0.001)$ on the basis of age, DBP as well as controls vs CKF $(p=\leq 0.001)$ on the basis of age only not the DBP. There was also significant association between controls vs IN $(p=0.005)$; controls vs ON $(p=\leq 0.001)$; controls vs CKF $(\mathrm{p}=\leq 0.001)$ on the basis of SBP.

Further, in case of biochemical parameter using same analysis assuming that in T1DM, there was a significant association between controls vs PN $(p=\leq 0.001)$, controls vs IN, $(p=\leq 0.001)$, control vs $\mathrm{ON}(\mathrm{p}=\leq 0.001)$ on the basis of FBS. There was also a significant association between controls vs PN $(p=0.014)$ on the basis of eGFR and between controls and IN $(p=0.001)$ on the basis of sCreatinine. 
Proportion of diabetic nephropathy, Bangladesh

Table 2 Association of physiological and biochemical profiles with DN stages in T1DM and T2DM patients

\begin{tabular}{|c|c|c|c|c|c|}
\hline \multicolumn{6}{|c|}{ Physiological profiles and DN stages } \\
\hline Groups & Study Subjects & Age (years) & $\mathrm{BMI}\left(\mathrm{kg} / \mathrm{m}^{2}\right)$ & SBP (mm Hg) & $\mathrm{DBP}(\mathrm{mm} \mathrm{Hg})$ \\
\hline \multirow[t]{12}{*}{ T1DM } & Control $(\mathrm{n}=50)$ & $29.98 \pm 7.7$ & $23.76 \pm 2.5$ & $118.20 \pm 6.3$ & $81.2 \pm 4.7$ \\
\hline & Pre-nephropathy $(n=4)$ & $21.00 \pm 1.5$ & $23.08 \pm 1.80$ & $120.00 \pm 8.2$ & $81.25 \pm 6.3$ \\
\hline & $\begin{array}{l}\text { Incipient nephropathy } \\
(\mathrm{n}=33)\end{array}$ & $22.94 \pm 2.5$ & $21.76 \pm 2.8$ & $121.21 \pm 8.9$ & $82.21 . \pm 5.8$ \\
\hline & $\begin{array}{l}\text { Overt nephropathy } \\
(n=3)\end{array}$ & $21.33 \pm 1.5$ & $23.02 \pm 1.4$ & $123.00 \pm 5.7$ & $83.67 \pm 2.8$ \\
\hline & $\operatorname{ANOVA}\left(p^{*}\right.$ Value $)$ & $\leq 0.001$ & 0.012 & 0.080 & 0.069 \\
\hline & Multiple Comparisons & \multicolumn{4}{|c|}{ post-hoc LSD } \\
\hline & Controls vs., PN & 0.005 & 0.619 & 0.591 & 0.332 \\
\hline & Controls vs., IN & $\leq 0.001$ & 0.001 & 0.040 & 0.029 \\
\hline & Controls vs., ON & $\leq 0.001$ & 0.635 & 0.206 & 0.295 \\
\hline & PN vs.,IN & 0.770 & 0.343 & 0.723 & 0.986 \\
\hline & PN vs., ON & 0.943 & 0.974 & 0.178 & 0.141 \\
\hline & IN vs.,ON & 0.868 & 0.428 & 0.045 & 0.066 \\
\hline \multirow[t]{17}{*}{ T2DM } & Control $(\mathrm{n}=50)$ & $29.98 \pm 7.7$ & $23.76 \pm 2.5$ & $118.20 \pm 6.3$ & $81.20 \pm 4.7$ \\
\hline & Pre-nephropathy $(n=11)$ & $56.18 \pm 10.4$ & $24.63 \pm 2.10$ & $134.55 \pm 12.1$ & $84.18 \pm 4.0$ \\
\hline & $\begin{array}{l}\text { Incipient nephropathy } \\
(n=162)\end{array}$ & $54.33 \pm 9.1$ & $23.76 \pm 3.52$ & $134.71 \pm 15.7$ & $84.34 \pm 6.0$ \\
\hline & $\begin{array}{l}\text { Overt nephropathy } \\
(\mathrm{n}=20)\end{array}$ & $57.50 \pm 9.3$ & $24.30 \pm 5.08$ & $136.50 \pm 17.8$ & $86.75 \pm 7.6$ \\
\hline & CKF $(n=7)$ & $62.71 \pm 10.7$ & $23.86 \pm 2.55$ & $140.00 \pm 20.8$ & $88.80 \pm 7.6$ \\
\hline & ANOVA(p* Value) & $\leq 0.001$ & 0.862 & $\leq 0.001$ & $\leq 0.001$ \\
\hline & Multiple Comparisons & \multicolumn{4}{|c|}{ post-hoc LSD } \\
\hline & Controls vs. PN & $\leq 0.001$ & 0.592 & 0.001 & $\leq 0.001$ \\
\hline & Controls vs. IN & $\leq 0.001$ & 0.617 & $\leq 0.001$ & $\leq 0.001$ \\
\hline & Controls vs. ON & $\leq 0.001$ & 0.281 & $\leq 0.001$ & $\leq 0.001$ \\
\hline & Controls vs. CKF & $\leq 0.001$ & 0.946 & $\leq 0.001$ & 0.106 \\
\hline & PN vs. IN & 0.508 & 0.753 & 0.619 & 0.028 \\
\hline & PN vs. ON & 0.696 & 0.776 & 0.717 & 0.495 \\
\hline & PN vs. CKF & 0.134 & 0.754 & 0.434 & 0.050 \\
\hline & IN vs. ON & 0.137 & 0.388 & 0.220 & 0.070 \\
\hline & IN vs. CKF & 0.016 & 0.890 & 0.167 & 0.492 \\
\hline & ON vs CKF & 0.187 & 0.556 & 0.580 & 0.113 \\
\hline \multicolumn{6}{|c|}{ Biochemical profiles and DN stages } \\
\hline & Study subjects & $\begin{array}{r}\text { FBS } \\
(\mathrm{mmol} / \mathrm{L})\end{array}$ & $\begin{array}{r}\text { sCreatinine } \\
(\mu \mathrm{mol} / \mathrm{L})\end{array}$ & $\begin{array}{r}\text { eGFR* } \\
(\mathrm{mL} / \mathrm{min})\end{array}$ & $\begin{array}{r}\mathrm{eGFR} * * \\
((\mathrm{~mL} / \mathrm{min})\end{array}$ \\
\hline \multirow[t]{12}{*}{ T1DM } & Control $(\mathrm{n}=50)$ & $4.55 \pm 0.52$ & $61.46 \pm 6.86$ & $110.54 \pm 13.2$ & $109.22 \pm 10.84$ \\
\hline & Pre-nephropathy $(n=4)$ & $7.55 \pm 0.85$ & $61.98 \pm 6.10$ & $92.25 \pm 10.3$ & $83.00 \pm 37.69$ \\
\hline & $\begin{array}{l}\text { Incipient nephropathy } \\
(\mathrm{n}=33)\end{array}$ & $7.78 \pm 0.79$ & $67.10 \pm 8.39$ & $90.18 \pm 14.8$ & $41.45 \pm 13.28$ \\
\hline & $\begin{array}{l}\text { Overt nephropathy } \\
(\mathrm{n}=3)\end{array}$ & $7.83 \pm 1.41$ & $67.86 \pm 10.86$ & $82.54 \pm 15.6$ & $78.58 \pm 14.50$ \\
\hline & ANOVA (p* Value) & $\leq 0.001$ & 0.004 & 0.037 & 0.028 \\
\hline & Multiple Comparison & \multicolumn{4}{|c|}{ Post hoc LSD } \\
\hline & Controls vs., PN & $\leq 0.001$ & 0.087 & 0.014 & 0.08 \\
\hline & Controls vs., IN & $\leq 0.001$ & 0.001 & 0.699 & 0.455 \\
\hline & Controls vs., ON & $\leq 0.001$ & 0.528 & 0.152 & 0.145 \\
\hline & PN vs.,IN & 0.017 & 0.785 & 0.026 & 0.023 \\
\hline & PN vs., ON & 0.048 & 0.098 & 0.006 & 0.005 \\
\hline & IN vs.,ON & 0.681 & 0.064 & 0.116 & 0.170 \\
\hline \multirow[t]{2}{*}{ T2DM } & Control $(\mathrm{n}=50)$ & $4.55 \pm 0.58$ & $61.46 \pm 6.86$ & $110.54 \pm 13.2$ & $109.22 \pm 10.84$ \\
\hline & $\begin{array}{l}\text { Pre-nephropathy } \\
(\mathrm{n}=11)\end{array}$ & $7.84 \pm 3.20$ & $77.78 \pm 25.16$ & $92.73 \pm 26.4$ & $90.88 \pm 28.22$ \\
\hline
\end{tabular}




\begin{tabular}{|c|c|c|c|c|}
\hline $\begin{array}{l}\text { Incipient nephropathy } \\
(n=162)\end{array}$ & $8.26 \pm 3.93$ & $77.78 \pm 21.35$ & $75.51 \pm 19.9$ & $73.99 \pm 25.66$ \\
\hline $\begin{array}{l}\text { Overt nephropathy } \\
(n=20)\end{array}$ & $8.28 \pm 3.17$ & $79.30 \pm 24.40$ & $73.40 \pm 28.8$ & $70.90 \pm 29.89$ \\
\hline $\operatorname{CKF}(n=7)$ & $8.48 \pm 4.26$ & $190.63 \pm 63.29$ & $22.14 \pm 4.7$ & $19.78 \pm 4.44$ \\
\hline ANOVA ( $p^{*}$ Value) & $\leq 0.001$ & $\leq 0.001$ & $\leq 0.001$ & 0.001 \\
\hline Multiple Comparison & \multicolumn{4}{|c|}{ Post hoc LSD } \\
\hline Controls vs. PN & $\leq 0.001$ & 0.001 & $\leq 0.001$ & 000.1 \\
\hline Controls vs. IN & $\leq 0.001$ & $\leq 0.001$ & $\leq 0.001$ & $\leq 0.001$ \\
\hline Controls vs. ON & $\leq 0.001$ & 0.003 & $\leq 0.001$ & $\leq 0.001$ \\
\hline Controls vs. CKF & $\leq 0.001$ & $\leq 0.001$ & $\leq 0.001$ & $\leq 0.001$ \\
\hline PN vs. IN & 0.789 & 0.185 & 0.032 & $\leq 0.001$ \\
\hline PN vs. ON & 0.480 & 0.370 & 0.135 & 0.234 \\
\hline PN vs. CKF & 0.655 & $\leq 0.001$ & $\leq 0.001$ & $\leq 0.001$ \\
\hline IN vs. ON & 0.142 & 0.748 & 0.640 & 0.444 \\
\hline IN vs. CKF & 0.730 & $\leq 0.001$ & $\leq 0.001$ & $\leq 0.001$ \\
\hline ON vs CKF & 0.273 & $\leq 0.001$ & $\leq 0.001$ & 0.001 \\
\hline
\end{tabular}

Results are expressed as Mean \pm SD. Statistical comparison of variables among age groups performed using One-Way ANOVA followed by LSD post hoc analysis. A 'p' value $<0.05$ was considered as statistically significant.

FBG Fasting Blood Glucose; sCreatinine Serum Creatinine; eGFR; Estimated Glomerular filtration Rate; BMI Body Mass Index; SBP: Systolic Blood Pressure; DBP: Diastolic Blood Pressure; PN Pre-Nephropathy; IN Incipient Nephropathy; ON Overt Nephropathy, CKF Chronic Kidney failure; T1DM Type 1 diabetes mellitus,; T2DM Type 2 diabetes mellitus

In case of $\mathrm{T} 2 \mathrm{DM}$, different stages of nephropathy have a significant association $(\mathrm{p}=\leq 0.001, \leq 0.001$, and $\leq 0.001)$ with FBS $(\mathrm{mmol} / \mathrm{L})$, screatinine $(\mu \mathrm{mol} / \mathrm{L})$ and $\quad$ eGFR $\left(\mathrm{mL} / \mathrm{min} / 1.73 \mathrm{~m}^{2}\right)$ (Table 2). From the post- hoc LSD multiple comparison test it was assumed that there was a significant association between controls vs PN $(\mathrm{p}=\leq 0.001, \quad 0.001, \leq 0.001)$, controls vs $\mathrm{IN}$, $(p=\leq 0.001, \leq 0.001, \leq 0.001)$, control vs ON $(p=\leq 0.001,0.003, \leq 0.001)$ and controls and CKF $(p=\leq 0.001, \leq 0.001, \leq 0.001)$ on the basis of FBS, sCreatinine and eGFR. PN vs IN ( $p=0.032)$ show significance association on the basis sCreatinine only. IN vs CKF ( $\mathrm{p}-\leq 0.001,0.003)$ and $\mathrm{ON}$ vs CKF $(p=\leq 0.001, \leq 0.001)$ show significant association on the basis of sCreatinine and eGFR. But in case of FBS $(p=0.789,0.480,0.655,0.142,0.730,0.273$ ) does not show significant association in different stages of DN.

Table 3 Factor associated with diabetic nephropathy (Crude and adjusted risk)

\begin{tabular}{|c|c|c|c|c|c|}
\hline Factors & & $\mathrm{COR}(95 \% \mathrm{CI})$ & P-value & $\operatorname{AOR}(95 \% \mathrm{CI})$ & P-value \\
\hline \multirow[t]{3}{*}{ Age (yrs) } & $60-79$ & $2.94(0.82-10.44)$ & & $1.05(1.01-1.08)$ & $0.023^{*}$ \\
\hline & $40-59$ & $1.88(0.46-7.49)$ & $0.04 *$ & $2.33(2.01-2.99)$ & $0.03 *$ \\
\hline & [20-39-Ref] & & & & \\
\hline Sex & $\begin{array}{r}\text { Female } \\
\text { [Male-Ref] }\end{array}$ & $1.89(0.86-4.65)$ & $0.03 *$ & $0.39(0.19-0.77)$ & $0.009 *$ \\
\hline \multirow[t]{3}{*}{ BMI $\left(\mathrm{kg} / \mathrm{m}^{2}\right)$} & $18.5-22.9$ & $3.75(0.67-9.92)$ & 0.44 & $3.45(0.55-8.80)$ & 0.20 \\
\hline & $23.0-24.9$ & $2.76(0.98-15.60)$ & 0.30 & $2.47(0.48-13.8 .76)$ & 0.22 \\
\hline & $\begin{array}{r}\geq 25.0 \\
5<18 \text {-Ref }\end{array}$ & $2.11(01.09-7.89)$ & 0.11 & $1.78(0.667-9.76)$ & 0.77 \\
\hline SBP & $\begin{array}{l}\text { Hypertensive } \\
\text { [Normal-Ref] }\end{array}$ & $1.03(1.01-1.05)$ & $0.01^{*}$ & $1.02(0.99-1.06)$ & 0.19 \\
\hline DBP & $\begin{array}{l}\text { Hypertensive } \\
\text { [Normal-Ref] }\end{array}$ & $1.08(1.02-1.15)$ & $0.007^{*}$ & $1.06(0.98-1.15)$ & 0.13 \\
\hline FBS (mmol/L) & $\begin{array}{r}\quad>6.1 \\
{[\leq 6.1-\operatorname{Ref}]}\end{array}$ & $0.68(0.40-1.96)$ & 0.33 & $0.88(0.39-1.55)$ & 0.45 \\
\hline
\end{tabular}

BMI Body Mass Index; FBS: Fasting Blood Sugar, SBP Systolic Blood Pressure; DBP; Diastolic Blood Pressure; *statistically significant at $<0.05$ significance level.

Irrespective of group, by univariate analysis, older age $(\mathrm{p}=0.04)$, sex $(\mathrm{p}=0.03)$, SBP $(\mathrm{p}=0.01)$ and DBP $(\mathrm{p}=0.007)$ were found to be significantly associated with diabetic nephropathy in diabetic patients. In multivariate analysis, older age (adjusted OR $=1.05$, CI: 1.01-1.08; adjusted OR: 2.33, CI: 2.01-2.99), and female sex (adjusted OR = 0.39 , CI: 0.19-0.77) were independently associated with DN adjusting BMI, SBP, DBP and FBS level (Table 03). 


\section{DISCUSSION}

Both T1DM and T2DM patients are affected by DN. Most patients with DN remain asymptomatic in early stages. Diagnosis of DN in diabetic patients warrants significant changes in management of patients both for DM and possible cardiovascular risk. ${ }^{10}$ The microalbuminuria is a marker for $\mathrm{DN}$ in patients with T1DM and T2DM. The present study reported the mean age of the T1DM, T2DM patient and control was $21.8 \pm 2.3$ years, $55.04 \pm 9.4$ years and $29.98 \pm 7.7$ years respectively. These characteristics were comparable with the study conducted in Pakistan (mean age of T2DM and controls were $57 \pm 8.12$ years and $52.6 \pm 9.58$ years respectively). ${ }^{11}$

In our study in T1DM the highest frequency was microalbuminuria or IN $(82.5 \%)$ followed by pre-nephropathy (10\%) and ON (7.5\%). Similarly, the highest frequency was microalbuminuria or IN $(81 \%)$ in those with T2DM followed by $\mathrm{ON}(10 \%)$, PN (5.5\%) and chronic kidney failure $(3.5 \%)$. A study conducted in India reported that the proportion rate of $\mathrm{ON}$ was $2.5 \%$ and microalbuminuria was $13 \%$ where $n=200 .{ }^{12} \mathrm{~A}$ similar study conducted in Korea reported proportion rate of albuminuria was $15.5 \%$ (microalbuminuria, 14.6\%; macroalbuminuria, $0.9 \%$ ) and $30.5 \%$ (microalbuminuria, $25.1 \%$; macroalbuminuria, 5.5\%) in subjects with prediabetes and diabetes, respectively. Another study reported the proportion rate of IN was $51 \%$ $(n=100)$ inT2DM. ${ }^{13}$ Yarasani conducted a study in 2014 found that $46 \%$ of the diabetic patients had IN. ${ }^{14}$ Benjamin \& et al. in their study, found that $43.1 \%$ of the diabetic patients had IN. A case-control study was done at out-patient department of BIRDEM General Hospital, Dhaka, Bangladesh reported one third of the study subjects had DN out of hundred. ${ }^{8}$

Our study revealed that there was significant association between different stages DN and mean of age, SBP, DBP $(\mathrm{p}=\leq 0.001 ; \leq 0.001$; $\leq 0.001$ ); but no significant association between DN stages and mean of BMI, $(p=0.599)$ in T2DM groups. Similarly in T1DM group there was significant association between DN stages and mean of age, BMI ( $p=0.001 ; 0.012)$, but no significant association between DN stages and mean of SBP and DBP ( $\mathrm{p}=0.800 .069) .{ }^{15,16}$ A study conducted in Bangladesh reported family history of DM and DN, higher BMI, presence of hypertension, and diabetic retinopathy were significant risk factors for $\mathrm{DN}$.

The mean age, BMI, FBS, serum creatinine, eGFR was significantly lower $(\mathrm{p}=\leq 0.001 ; 0.012 ; \leq 0.001 ; 0.004 ; .037 ;$ wherep $<$ 0.05 is considered statistically significant) in those with IN and $\mathrm{ON}$ and PN among T1DM in comparison with controls but there was no significant difference in SBP $(p=0.591)$ and DBP $(p=0.332)$. The mean age, FBS, SBP, DBP, sCreatinine, was significantly higher $(p=\leq 0.001$; $\leq 0.001 ; \leq 0.001 ; \leq 0.001$ where $\mathrm{p}<0.05$ was considered statistically significant) in those with IN and $\mathrm{ON}$ and PN in T2DM in comparison with controls. A study conducted in Turkey revealed that IN had a highly significant correlation with mean of age and BMI $(\mathrm{p}<0.001){ }^{14}$

Further a study conducted in India found that the mean FBS levels, SBP was also high in type T2DM compared to controls $(\mathrm{p}<0.001) .{ }^{16}$ In our study FBS was significantly higher in the IN and ON group compared with the PN ( $p=0.017 ; 0.048)$. Yarasani study revealed that FBS was significantly higher in the IN compared with the PN $(p=0.02) .{ }^{14}$ Similar results were obtained in a study conducted by Varghese et.al. $(p<0.001)$. In our study, among T2DM the sCreatinine was significantly higher among those with stage of CKF than those in ON. Similar results were obtained in other study conducted by Varghese et al, Muhammad Baig et al, Benjamin A, Eghan et al. ${ }^{17,18}$

Our study had several limitations. This was a single center study cross-sectional study and included limited number of patients. A nationally representative multicenter prospective cohort study would have provided better evidence on the proportion of DN in Bangladesh. Both the case and control groups were selected using convenience sampling which limits generalizability. The present study categorized the patients in different nephropathy stages (ACR: $\geq 30 \mathrm{mg} / \mathrm{g}$ ) on the basis of one sample test only which resulted in overestimation or underestimation.

\section{CONCLUSION}

This study showed the proportion of $\mathrm{DN}$ was high among diabetic and mostly in T2D patients with severe stage. Irrespective of diabetic group, older age, and female sex were independently associated with DN. However, these findings underscore the need for intensifying diabetes education measures to the community at large and to diabetic subjects in particular. Imparting knowledge about diabetes to the community is the first step in prevention and early detection of the disease and prevention of its complications.

\section{Conflict of interest}

Authors have declared that no competing interests exist.

\section{ACKNOWLEDGEMENT}

We express our gratitude to MA Wahed for his continuous support in every steps of the study. We also express our acknowledgement to all the colleagues of Rangpur Diabetic Association and Apollo Diagnostic and Imaging Centre, Rangpur who helped us to collect data from the study subjects and to provide laboratory support for biochemical analysis. 
Authorship

'Dr. Md. Abdur Rahman', 'Dr. Md. Ruhul Forkan Siddique' and 'Most. Zannatul Ferdous' designed the study, Most. Zannatul Ferdous performed the statistical analysis, prepared the first draft of the manuscript. 'Md. Abdul Halim'. 'Most. Zannatul Ferdous' and 'Sheikh Jafia Jafrin' managed the literature searches. All authors of this paper have read and approved the final version submitted. The contents of this manuscript have not been copyrighted or published previously. The contents of this manuscript are not now under consideration for publication elsewhere. Additionally, all of the authors have agreed to the Journal of "International Journal of Public Health Research" submission policies.

\section{REFERENCES}

1. WHO. Diabetes Fact Sheet. NMH Fact Sheet Febr 2010. 2010. Available from: doi:10.3343/alm.2017.37.1.28.

2. World Health Organization. Diabetes: The Problem. 2008:1-2. Available from: https://www.who.int/nmh/publications/fact sheet_diabetes_en.pdf.

3. Jian W. Peng W. Jin J. et al. Metabolism(Press). Diabetes Care. 2011.

4. Farag YMK, Al Wakeel JS. Diabetic nephropathy in the arab gulf countries. Nephron - Clin Pract. 2011. Available from: doi:10.1159/000328909.

5. Sanz M, Ceriello A, Buysschaert M, et al. Scientific evidence on the links between periodontal diseases and diabetes: Consensus report and guidelines of the joint workshop on periodontal diseases and diabetes by the International diabetes Federation and the European Federation of Periodontology. In: Diabetes Research and Clinical Practice. 2018. Available from: doi:10.1016/j.diabres.2017.12.001.

6. Wen CP, Cheng TYD, Tsai MK, et al. Allcause mortality attributable to chronic kidney disease: a prospective cohort study based on 462293 adults in Taiwan. Lancet. 2008. Available from: doi:10.1016/S01406736(08)60952-6.

7. Chandie Shaw PK, Baboe F, Van Es LA, et al. South-Asian type 2 diabetic patients have higher incidence and faster progression of renal disease compared with Dutch-European diabetic patients. Diabetes Care. 2006. Available from: doi:10.2337/dc06-0003.

8. National Guideline Clearinghouse. Standards of medical care in diabetes. V. Diabetes care. Natl Guidel Clear. 2013;1:S11-66. Available from: http://www.guideline.gov/content.aspx?id $=45153 \&$ search $=$ diabetes .
9. Nah EH, Cho S, Kim S, Cho HI. Comparison of urine albumin-to-creatinine ratio (ACR) between acr strip test and quantitative test in prediabetes and diabetes. Ann Lab Med. 2017;37(1):28-33. Available from: doi:10.3343/alm.2017.37.1.28.

10. MA R, Zaman S, Habib SH, Afsana F, Haque WM and Iqbal S. Evaluation of risk factors for diabetic nephropathy among newly diagnosed type 2 diabetic subjects: preliminary report from a tertiary care hospital of Bangladesh. BIRDEM Medical Journal. 2020; 10 (2): 88-91. [cited 2020 Jun] Available from:https://doi.org/10.3329/birdem.v10i2 .47732 .

11. Haneda M, Utsunomiya K, Koya D, et al. A new Classification of Diabetic Nephropathy 2014: A report from Joint Committee on Diabetic Nephropathy. J Diabetes Investig. 2015. Available from: doi:10.1111/jdi.12319.

12. Collins AJ, Li S, Gilbertson DT, Liu J, Chen S-C, Herzog CA. Chronic kidney disease and cardiovascular disease in the Medicare population. Kidney Int. 2003;64:S24-S31. Available from: doi:10.1046/j.1523-1755.64.s87.5.x.

13. Hussain F, Maan M, Medical MF-BJ of, 2010 U. Plasma protein glycation status in Pakistan type 2 diabetic patients with or without nephropathy. BanglajolInfo. 2010;9(2):68-75. Available from: https://www.banglajol.info/index.php/BJM S/article/view/5654.

14. Vimalkumar VK, Moses CA, Padmanaban S. Proportion \& Risk Factors of Nephropathy in Type 2 Diabetic Patients. Int J Collab Res Intern Med Public Heal. 2011;3(8):598-615.

15. Dayanidhi S, Ramadevi K, Periyandavar I. Prevalance and predictors of microalbuminuria in patients with type 2 diabetes mellitus. Int $\mathrm{J}$ Pharma Bio Sci. 2013;4(4).

16. Yarasini A. Microalbuminuria As a Marker of Cardiovascular and Renal Risk in Type Ii Diabetes Mellitus. J Evol Med Dent Sci. 2014;3(28):7701-7707. Available from: doi:10.14260/jemds/2014/2959.

17. Preity S, Delwer M, Hawlader H, Akhter S, Abdullah AS, Biswas A. Views of the Parents of Autistic Children about Autism and Schools for Autistic Children: A Qualitative Study in Urban Bangladesh. Int J Public Heal Res. 2017;5(5):56-61.

18. Jyothirmayi B. Study of microalbumin in type 2 diabetes mellitus with less than and more than five years of duration. Int $\mathrm{J}$ 
Proportion of diabetic nephropathy, Bangladesh

Pharma Bio Sci. 2014. 